\title{
Scanning Optical Head with Nontilted Reference Beam: Assuring Nanoradian Accuracy for a New Generation Surface Profiler in the Large-Slope Testing Range
}

\author{
Shinan Qian \\ Instrumentation Division, Brookhaven National Laboratory, Upton, NY 11973, USA \\ Correspondence should be addressed to Shinan Qian, qian@bnl.gov \\ Received 12 October 2010; Accepted 28 March 2011 \\ Academic Editor: Wolfgang Osten \\ Copyright (C) 2011 Shinan Qian. This is an open access article distributed under the Creative Commons Attribution License, which \\ permits unrestricted use, distribution, and reproduction in any medium, provided the original work is properly cited.

\begin{abstract}
Nanoradian Surface Profilers (NSPs) are required for state-of-the-art synchrotron radiation optics and high-precision optical measurements. Nano-radian accuracy must be maintained in the large-angle test range. However, the beams' notable lateral motions during tests of most operating profilers, combined with the insufficiencies of their optical components, generate significant errors of $\sim 1 \mu \mathrm{rad} \mathrm{rms}$ in the measurements. The solution to nano-radian accuracy for the new generation of surface profilers in this range is to apply a scanning optical head, combined with nontilted reference beam. I describe here my comparison of different scan modes and discuss some test results.
\end{abstract}

\section{Nanometer and Nanoradian Accuracy Requirements for Ultraprecise Mirrors and Their Metrology}

Hard X-rays of synchrotron radiation (SR) are very important for chemical, elemental, and structural analyses of matter at the nano- and atomic scale and for elucidating the molecular processes involved in biological functions at the cellular level. Scientists anticipate having one-nanometer probe spots for such research.

Recently, construction started on ultrabright SR sources with nanofocusing spots at the National Synchrotron Light Source II (NSLS II), Brookhaven National Laboratory (BNL) in the United States and at the Taiwan Photon Source (TPS) of the NSSRC in Taiwan. The NSLS II will allow researchers to create high-contrast X-ray images of matter at this resolution.

To focus the bright, hard X-rays of an SR beam into $1 \mathrm{~nm}$ spot, the beam lines must incorporate a series of precise optics. One of the most promising approaches to do so is applying a total reflection mirror with its exceptional characteristics of broadband, achromaticity, and high efficiency. Japanese researchers broke the 10-nm barrier in hard X-ray focusing on one direction, realizing a width of $7 \mathrm{~nm}$ at $20 \mathrm{keV}$ using a multiple-layered mirror [1]. However, the mirror figure employed to focus an SR beam to a nanometer spot while preserving coherence should have nanoradian (nrad) accuracy. According to simple geometric-optics calculations, if the error in surface slope is $100 \mathrm{nrad}$, the beam will exhibit a lateral displacement of $4 \mathrm{~nm}$ at a $20 \mathrm{~mm}$ focal distance; hence, it will greatly enlarge the $1 \mathrm{~nm}$ spot.

In addition, X-ray free electron lasers (XFELs) and extreme ultraviolet lithography (EUVL) demand a variety of precise optics with nanoradian accuracy.

Nanoradian accuracy is not only required for the lowspatial frequency region (figure). It could be much more crucial for mid-spatial frequencies because it entails a larger slope error, thereby blurring the image's quality and enlarging the focus point [2-4]. For example, one $20 \mathrm{~mm}$ sine wavefront $(1 \mathrm{~Hz})$ with 1 nanometer $(\lambda / 633)$ amplitude will produce $\pm 0.314 \mu \mathrm{rad}$ error in slope. However, if the sine ripple is $5 \mathrm{~Hz}$ ( $4 \mathrm{~mm}$ period) with the same amplitude, the slope error will increase to $\pm 1.57 \mu \mathrm{rad}$. This ripple is frequently evident from precisely polished surfaces. Modern subaperture and deterministic optical fabrication techniques are more prone to leave residual periodic surface 
undulations. Therefore, the best specification of precise surface error is to define its slope error at both high- and mid-spatial frequencies.

Undertaking precise metrological studies is the basic approach to fabricating optics. The accuracy of metrology should be at least twofold $(<50 \mathrm{nrad})$ better than the specifications for the optics' accuracy. The great challenge to metrology and manufacturing is attaining nanoradian accuracy for spheric- or aspheric-mirrors with a large surface-slope angle. The requirement is far beyond current possibilities in both these areas.

I summarize below the four nanoradian (nano) accuracy requirements for new-generation surface profilers mainly used for synchrotron radiation optics measurements:

(1) tests of in plane mirrors, including their nanostability and nano-repeatability;

(2) tests in the range of larger slopes, from $\pm 5 \mathrm{mrad}$ to $\pm 10 \mathrm{mrad}$;

(3) slope error tests in mid-spatial frequency (up to $0.1 \mathrm{~mm}$ ) for comparison to the recent $1-2 \mathrm{~mm}$ spatial frequency test, we expect to cover a large frequency range with the surface profiler, or develop another one in future (not detailed in this paper);

(4) absolute accuracy without removing any polynomial order.

\section{Recent Developments in the Accuracy of Nanoradian Measurements}

The traditional null interferometer can measure a 2D sphere or aspheric surface in full aperture with an accuracy of about $\lambda / 30$. However, the error in the reference mirror of a null lens limits the precision of the test. Making a null lens is time consuming and expensive, especially one for testing a large-aperture optical surface. Here, the computergenerated holograph $(\mathrm{CGH})$ is a good replacement for the traditional null system to reduce costs and preparation time. The CGH can test a broad range of asphericities [5]. In both cases, the measurement accuracy should be improved further and calibrated so that it fulfills the nanoradian accuracy requirement for the SR optics, EUV objective, and the freeelectron laser. The newly developed subaperture stitching interferometer enables the exact analysis of larger apertures, and aspheres can be measured without using dedicated large null optics [6, 7]. By automatically combining multiple subaperture measurements traceable to form a full-aperture measurement, many surfaces, formerly difficult to evaluate, are measurable quickly and affordably. Because stitching analysis might remove certain systematic errors, the SSI enhances the accuracy of measurements. However, stitching methods accumulate small systematic interferometer errors that limit their high accuracy. Hence, the accuracy of measurements of a full-size surface needs to be calibrated precisely [8]. Synchrotron-radiation optics adopt planes, spheres, and aspheres including cylinders, toroids, parabolics, and ellipsoids up to 1.5 meters long, operating in a grazing incidence angle. These lengthy cylindrical-like
TABLE 1: Different requirements for the slope error of mirrors and the focusing-spot size at different times.

\begin{tabular}{lcc}
\hline Years & $\begin{array}{c}\text { Slope error requirement } \\
\text { of mirrors }\end{array}$ & $\begin{array}{c}\text { Required focusing spot } \\
\text { size }\end{array}$ \\
\hline $1980 \mathrm{~s}$ & $5 \mu \mathrm{rad}$ & $10 \mu \mathrm{m}$ \\
$1990 \mathrm{~s}$ & $1 \mu \mathrm{rad}$ & $1 \mu \mathrm{m}$ \\
NSLS & $100 \mathrm{nrad}$ & $1 \mathrm{~nm}$ \\
$\begin{array}{l}\text { II/TPS and } \\
\text { new NSP }\end{array}$ & & \\
\hline
\end{tabular}

aspheric surfaces normally have a short radius of curvature, of tens millimeters sagittally, and several hundred meters or much more meridianly. Hence, they are hard to measure with a traditional null interferometer, a CGH or an SSI. A different kind of metrology was developed to suit the requirements for nanometer and nanoradian accuracy. A precise coordinate measuring machine is a good solution, opening up the possibility to assess the height of steep surfaces at the nanometer level in 2D [9]. Nevertheless, this is a contact test and probably during the test it will leave a very slight mark on the final surface. Now, pencil beam- scanning profilers are a promising noncontact method for large SR optics tests with nanoradian accuracy.

The usage of subaperture interferometer stitching method is starting for LTP- and SR-optics measurements $[10,11]$ with linear scanning. It will extend large optics tests into the 2D and mid-spatial frequency regions. However, improvements are needed in the accuracy of stitching, along with research on precise calibration methods.

The Long Trace Profiler (LTP) [12, 13], based on the principle of the pencil-beam interferometer [14], is designed for the metrology of second generation synchrotron radiation facilities. Its principle is similar to that underlying an autocollimator, but a pencil beam is employed to scan the mirror being tested. The scanning beam, deflected by $2 \alpha$ due to the local slope, $\alpha$, at the scanning point, is focused onto the position of $d=f * \tan (2 \alpha)$ on the CCD camera. In this way, we can reconstruct the whole curves of the slope and of the slope error of the scanned points. In the past 20 years, significant improvements made to the LTP satisfied the requirements of SR beam lines. From 1980s to the present, the requirement for slope error of SR optics rose from $5 \mu \mathrm{rad}$ to $100 \mathrm{nrad}$, while the needed focusing spot size on the beam line end station dropped to $1 \mathrm{~nm}$ from $10 \mu \mathrm{m}$ (Table 1).

Several LTPS were developed previously, including the following ones: the first LTP I, the commercial LTP II, the pentaprism LTP [15], the in situ LTP [16], the portable LTP [17], the vertical scan LTP [18] and the multiple functions LTP [19]. New metrology methods included the in situ heatload distortion test [20], and the in situ angle monitoring of the grating monochromator [21]. Furthermore, ESRF [22], SOLEIL [23], and SSRF [24] devised other LTPs.

Recently, researchers at BESSY II in Germany developed a Nano-Optic-Measuring Machine (NOM) [25], the most accurate instrument so far for evaluating SR optics. The uncertainty in the measurements of the NOM was low: for a plane mirror it was $0.05 \mu \mathrm{rad} \mathrm{rms}$, and, for curved mirror, it 


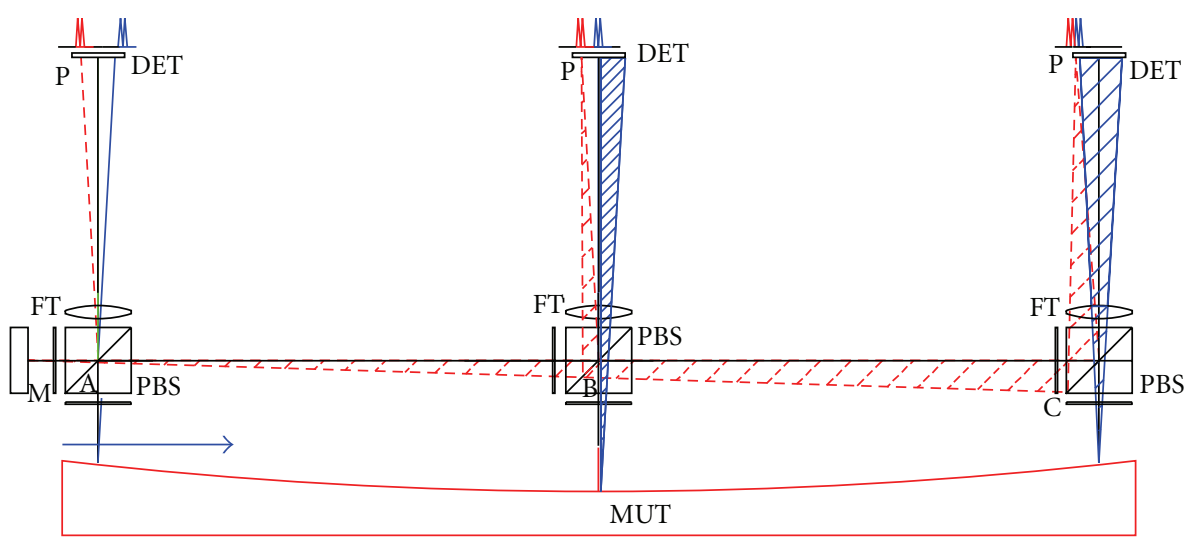

Figure 1: Sample and reference beams' lateral motion in scan optical head mode.

was $0.2 \mu \mathrm{rad} \mathrm{rms}$ [26]. The NOM combines, in a comparison test, an LTP and a commercial autocollimator with a small aperture. Both instruments operate in a scanning pentaprism mode without a reference beam. A similar Diamond-NOM was developed later [27].

A new traceable multiple sensor (TMS) system was developed recently for measuring with nanometer accuracy. It encompasses coupled multiple distance-sensors that are scanned along the surface under test. By using a small sensor head, a high lateral resolution is achieved [28-30]. In addition to the multiple distance sensors, the TMS utilizes an autocollimator measuring the tilt of the sensor's head, thereby eliminating systematic errors in the distance sensors. Seemingly, the TMS can reach nanometer or better accuracy with high lateral resolution.

Both the NOM and TMS are suitable for plane mirror measurements with nanoradian accuracy. However, accuracy becomes problematic when they are used for testing largeslope surfaces.

\section{Problem of Accuracy of Nanoradian Measurements: Lateral Motion of the Sample- and Reference-Beams over Imperfect Optical Components}

Previously, most work aiming towards 100-nanoradian accuracy focused on plane- and near-plane-mirrors. The precision required for measuring strongly curved surfaces remains unresolved.

François et al. evaluating the method "An LTP stitching procedure with compensation of instrument errors" commented that it has "...improved the agreement of the results down to $0.2 \mu \mathrm{rad}$ in slope, and $1.6 \mathrm{~nm}$ in height" for the \pm 5 mrad angle range test" [11].

However, $0.2-0.3 \mu \mathrm{rad}$ is the accuracy limitation on testing large-slope mirrors.

The main restrictions on accuracy are the beams' lateral motion in the optical system during the measurements, combined with the insufficient quality of the profiler's optical components. A laterally moving beam will pick up, as systematic errors, any significant local-slope errors at the $\mu \mathrm{rad}$ level in imperfect optical components. If they cannot be removed, or their numbers greatly decreased, nano-accuracy cannot be attained in range of large-slope tests. During tests on a plane mirror, the beam has no lateral motion; hence, here nrad accuracy is obtainable.

3.1. Detailed Explanation of Beam Lateral Motion (BLM). The LTP II applies a scanning optical head mode to scan a pencil beam for a surface-profile test. The scanning sample beam (solid line, Figure 1) tests the slope of a mirror (represented by a sphere in the figure), while the reference beam (dashed line) eliminates pitch error in the air bearing. To avoid the overlapping of the sample and reference beams on the CCD camera, the LTP II reference beam is tilted. During the scan, both beams display lateral motions over the optical components, as is evidenced in the shadowed area. The sample beam's lateral motion is unavoidable because it is an essential condition for testing the slope of curved mirrors however, we can reduce the extent of lateral motion in the sample beam, thereby lowering systematic error.

Scanning in the pentaprism mode (Figure 2) to test curved mirrors entails a larger BLM. This scanning mode is much more suitable for plane and near-plane mirror tests.

3.2. How Large Is the Slope Error Produced by a Tilted Reference Beam? We undertook sequential comparisons at BNL between the tilted reference method and the nontilted one on the reference arm of the LTP III in 900-mm scanning distance. In the first scan, the reference beam had a small 1.5-mrad tilt, equivalent to $3-\mathrm{mm}$ of lateral motion on the polarization beam-splitter (Figure 3(a)); the second scan was made without tilting (Figure 3(b)). Their difference (Figure 3(c)) of $\pm 5 \mu \mathrm{rad}(\mathrm{P}-\mathrm{V}$ ) constitutes a serious slope error for a nanoradian surface-profiler. When testing strongly curved mirror, the tilt angles in both the sample and reference beams increased, so that the systematic error of the LTP was severe.

An effective way to eliminate systematic error in the reference arm is to use a nontilted reference beam.

We also must remain aware of the impacts on the accuracy of assessing BLM caused by imperfect commercial optics. 


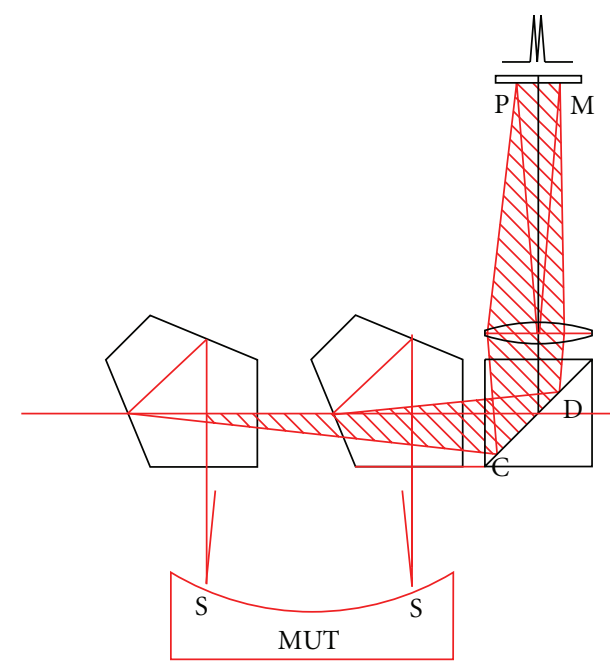

FIgURE 2: Beams' lateral motion on a scan in the pentaprism mode.

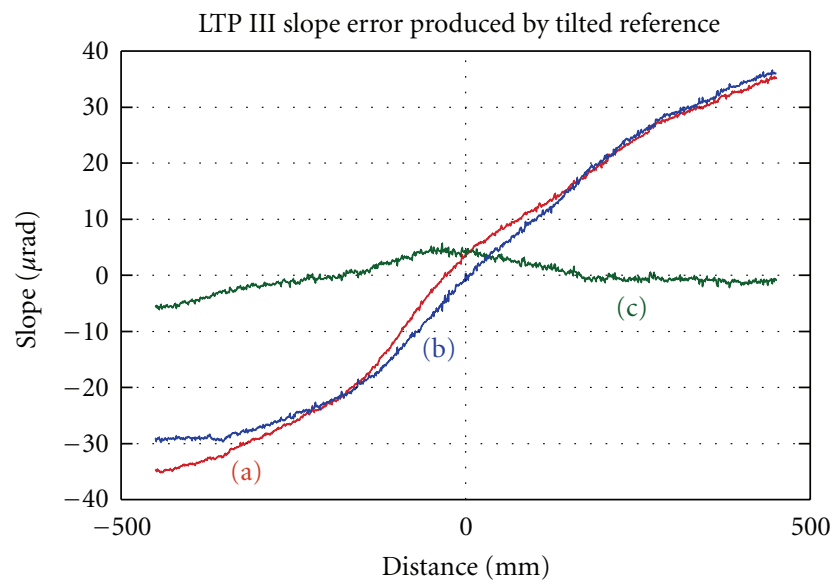

FIGURE 3: LTP III slope error produced by tilted reference. (a) Reference beam's spot is tilted in $1.5 \mathrm{mrad}$. (b) Nontilted reference beam at the center of the CCD. (c) Slope error caused by tilted reference.

3.3. Beam-Slope Error Produced by a Polarization Beam Splitter (PBS). The PBS is the main component of the LTP, set in front of the FT lens, that suffers from the BLM. Figure 4 shows our method of measuring the slope error induced by the BLM over the PBS by employing multiple functions LTP (LTP MF). For simplicity, the lens (FT) and detector (DET) represent the LTP MF in the figure; the PBS used in the LTP MF is not displayed. The LTP MF sends out a pencil beam that is reflected back to it by the mirror (M). During a stability scan of the LTP MF, the PBS being tested scans laterally over the pencil beam to simulate its BLM. The difference between stability curve obtained and a straight line is the double BLM slope error produced by the PBS.

Figure 5 shows the test results from a polarization beam splitter (PBS) made of commercial Homosil glass produced by Rainbow Research Optics Inc. with size of $25.4 \times 25.4 \times$ $12.7 \mathrm{~mm}$. Figure 6 displays the findings from tests of an

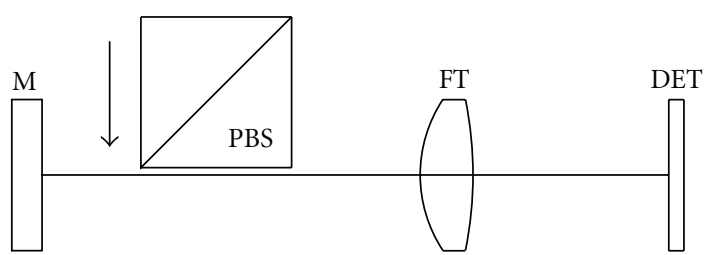

Figure 4: Slope error measurement of the PBS by the LTP MF (shown as FT lens and DET detector). The PBS scans through the test beam of the LTP MF to simulate the BLM.

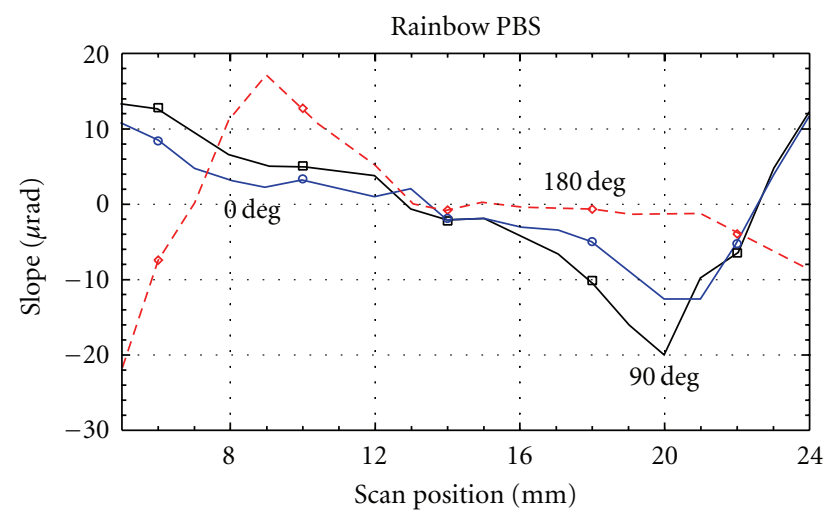

FIGURE 5: Results of slope measurement of the PBS produced by Rainbow Research Optics Inc., USA, tested at $0^{\circ}, 90^{\circ}$, and $180^{\circ}$ positions.

additional PBS used for the LTP MF, with a surface accuracy $1 / 10$ wavelength and an angle accuracy of 10 arc seconds, produced by Anhui Institute of Optics and Fine Mechanics, China, $20 \times 20 \times 20 \mathrm{~mm}$; the optical glass material is BK7. These measurements were acquired at the $0^{\circ}$ and $90^{\circ}$ (or additionally, at $180^{\circ}$ ) directions of the PBS. Both beam splitters revealed that there are slope errors of 3-4 $\mu \mathrm{rad} \mathrm{rms}$ with an aperture of $12 \mathrm{~mm}$ BLM. After detrending " 1 ", the error of the residual slope still is at about the $1.7 \mu \mathrm{rad}$ rms level for the large test range/large BLM (Figure 6). Because the test beam passes the PBS twice, the real slope error to impact accuracy of testing the LTP in single path is about $0.8-0.9 \mu \mathrm{rad}$ rms.

3.4. Slope Error Caused by the Beam Lateral Motion (BLM) for All Optical Systems. Figure 7 illustrates the method of measuring the slope error caused by the BLM for all LTP MF optical system. The CCD should be precisely positioned on the focal plane. During the stability scan of the LTP MF (PBS, FT and DET on Figure 7), an external pencil beam is scanned laterally to simulate the BLM over the LTP MF aperture by a pentaprism. If the LTP's optical system is perfect, all parallel beams will be focused to theoretical point. Hence, the slope curve will be a nonerror straight line. We obtained a slope error of $\sim 2 \mu \mathrm{rad}$ rms (Figure 8 ), caused by the BLM over the entire LTP MF system and the FT lens's aberration. The slope error of the BLM could reach $1 \mu \mathrm{rad}$ rms or more. Due to the characteristics of the pentaprism, theoretically all scanning 


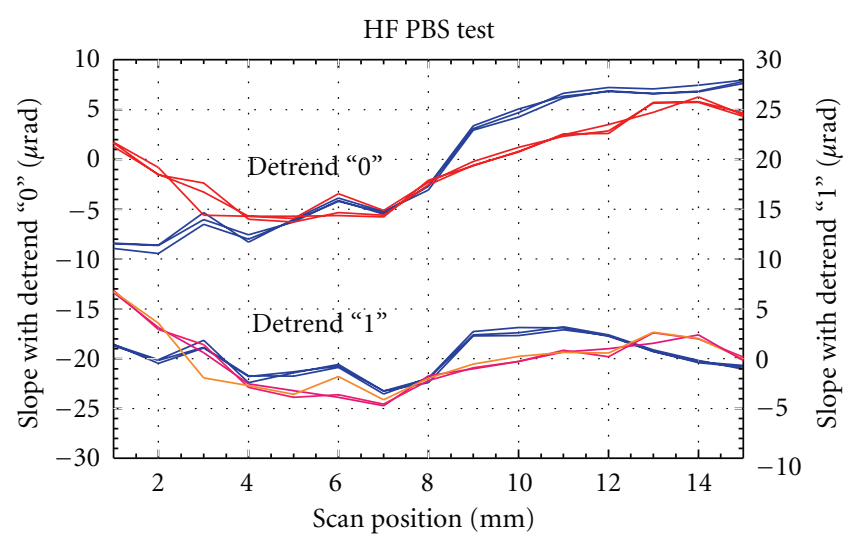

FIgure 6: Results of slope measurement of the PBS produced in China, tested at $0^{\circ}$ and $90^{\circ}$ positions. At each position, it is scanned three 3 times.

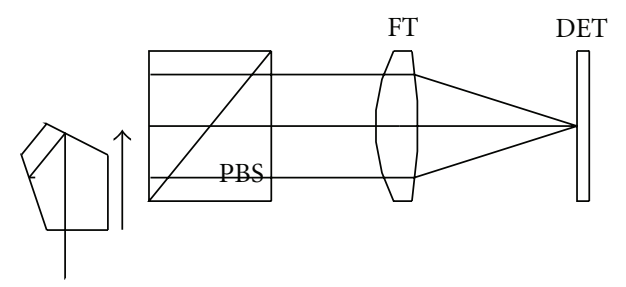

FIGURE 7: Method of measuring systematic slope error caused by the BLM for the LTP MF: an external pencil beam is scanned laterally over the LTP MF aperture by a pentaprism. If there is no systematic slope error, the slope curve should be a straight line.

beams are parallel, so a scanning pitch error of the pentaprism would not entail a slope error. To assess the slope error caused by the BLM in a tilted angle, it is necessary to rotate the LTP MF.

The surface figure errors and the inhomogeneity of optical components are the main sources generating a BLM slope error. From the test results, the value of the slope error caused by the BLM is about $1 \mu \mathrm{rad}$ rms or upwards into the large-slope range. Therefore, the quality of the optics must be improved greatly. However, high-quality optical components are difficult to fabricate. According to simulations, optical glass with grade $\mathrm{H} 5$ homogeneity and $\lambda / 100$ surface quality are needed [31] for entire aperture application. Even if these requirements are met, there still will be a gap before nanoradian accuracy is reached. More investigations are needed for fabricating a new nanoradian surface profiler (NSP).

3.5. Different Scan Modes Have Very Different Beam Lateral Motions. Table 2 compares three scan modes, highlighting the different BLMs.

According to this comparison, only the scan $\mathrm{OH}+$ nontilted REF (SO-NTR) mode has $\pm 0.5 \mathrm{~mm}$ BLM in the $\pm 5 \mathrm{mrad}$ test range; in contrast, the BLM of the scan pentaprism is 22 times larger.

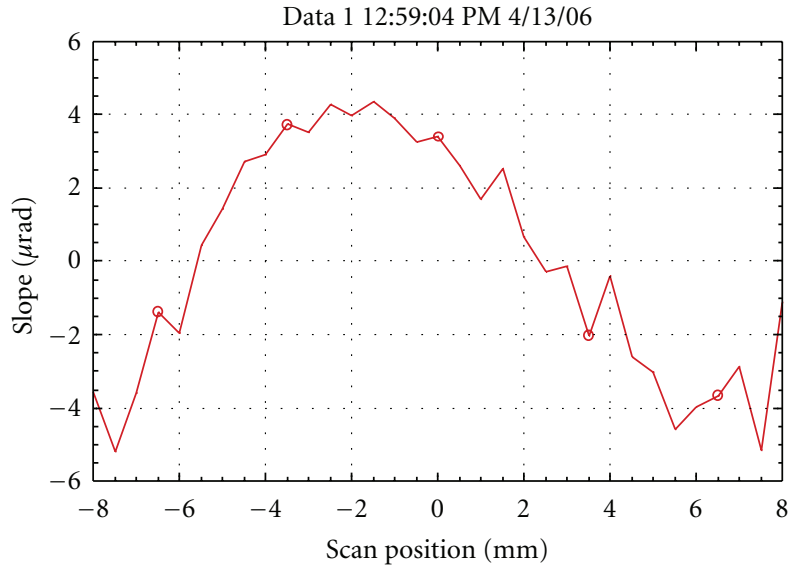

FIGURE 8: Systematic slope error caused by the BLM is $\sim 2 \mu \mathrm{rad} \mathrm{rms}$ for the LTP MF.

\section{Scanning Optical head Combined with Nontilted Reference Beam: Nanoradian Accuracy Solution for a New-Generation Surface Profiler in the Large-Slope Testing Range}

4.1. Advantages of a Scanning Optical Head (OH) Mode. As I described above, to ensure the nanoradian accuracy for a large test angle, a new nanoradian surface profiler (NSP) must apply a scanning optical head mode. The great advantage here is that the fixed short-working distance of the sample beam reduces the sample's BML to $\pm 0.5 \mathrm{~mm}$ that significantly lowers the systematic error in slope.

Other great advantages of applying a scanning optical head is the ease of calibration, and the compensation achievable for residual systematic errors because of the sample beam's fixed working distance. Furthermore, one compensation curve will suffice to correct all systematic errors in testing surfaces with different curvatures. In contrast, it is very hard to compensate for systematic errors in the pentaprism scan mode because surfaces with different curvatures tested at different distances necessitate applying different compensation curves [32]. Indeed, in the large test range, scanning in the pentaprism mode, almost precludes reaching nanoradian accuracy due to the BLM.

The third advantage is that the lower BLM and fixed working distance minimize the operational aperture, so simplifying the aberration-deduction designs of the lens.

4.2. Nontilted Reference Methods. Applying a nontilted reference beam will eliminate all lateral motion in the reference arm. Six methods are available for employing such a reference beam (Figure 9):

(a) using extra angle devices, namely, the autocollimator (BNL's LTP I) [33], a tiltmeter (ALS) [34], and others;

(b) sequentially measuring the sample and reference beams under the same conditions (at different times to preclude overlapping them); 


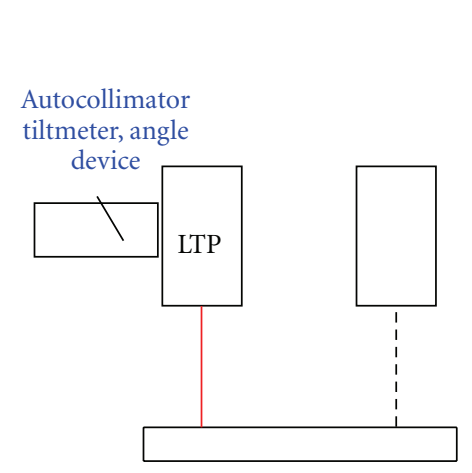

(a)

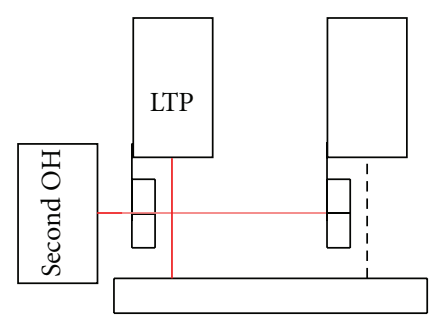

(d)

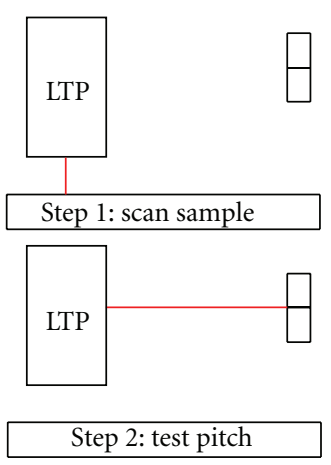

(b)

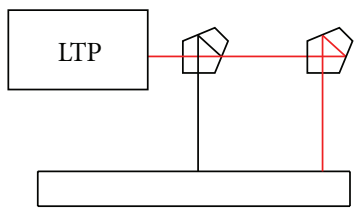

(e)

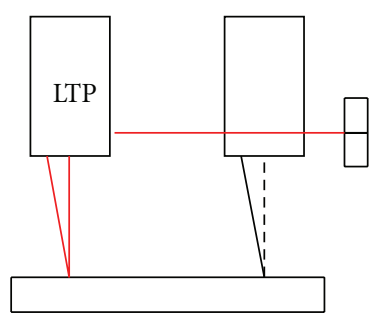

(c)

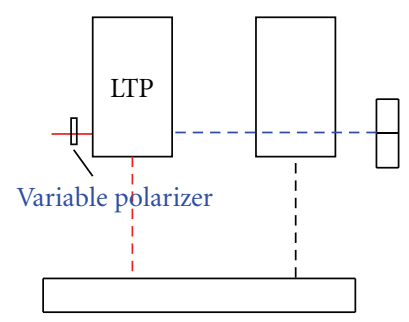

(f)

Figure 9: (a) Apply extra angle device to test pitch error; (b) take sample and reference beam tests in sequence; (c) use nontilted reference with tilted sample beam for plane mirror test; (d) employ second optical head; (e) assure non-reference beam by using pentaprism scan for plane mirror test; (f) alternatively switching on/off of sample and reference beams.

TABLE 2: Comparison of three scan modes.

\begin{tabular}{|c|c|c|c|c|c|}
\hline Scan mode & Working distance $(\mathrm{mm})$ & $\mathrm{BLM}(\mathrm{mm}) /$ at test angle $(\mathrm{mrad})$ & Test angle (mrad) & Extra optics & Comment \\
\hline \multirow{3}{*}{$\begin{array}{l}\text { Scan OH+ nontilted } \\
\text { REF (new NSP) }\end{array}$} & Sample: 50 & Sample: $\pm 0.5 \mathrm{~mm} / \pm 5$ & \multirow{3}{*}{ \pm 10} & \multirow{3}{*}{ N/A } & \multirow{3}{*}{$\begin{array}{l}\text { Larger test angle }+ \\
\text { high accuracy }\end{array}$} \\
\hline & (fixed) & $\pm 1 \mathrm{~mm} / \pm 10$ & & & \\
\hline & Ref: $100-1100$ & Ref: $0 \mathrm{~mm}$ & & & \\
\hline \multirow{4}{*}{$\begin{array}{l}\text { Scan OH+ tilted } \\
\text { REF (LTP II) }\end{array}$} & Sample: 50 & Sample: $\pm 0.5 \mathrm{~mm} / \pm 5$ & \multirow{4}{*}{ \pm 5} & \multirow{4}{*}{ N/A } & \multirow{4}{*}{$\begin{array}{l}\text { Suitable for plane and } \\
\text { near-plane mirror tests }\end{array}$} \\
\hline & (fixed) & $\pm 1 \mathrm{~mm} / \pm 10$ & & & \\
\hline & \multirow{2}{*}{ Ref: $100-1100$} & Ref: $\pm 11 \mathrm{~mm} / \pm 5$ & & & \\
\hline & & $\pm 22 \mathrm{~mm} / \pm 10$ & & & \\
\hline $\begin{array}{l}\text { Scan pentaprism } \\
\text { LTP (NOM) }\end{array}$ & Sample: $100-1100$ & $\begin{aligned} \text { Sample: } & \pm 11 \mathrm{~mm} / \pm 5 \\
& \pm 22 \mathrm{~mm} / \pm 10\end{aligned}$ & \pm 5 & Pentaprism & $\begin{array}{l}\text { Suitable for plane and } \\
\text { near-plane mirror test }\end{array}$ \\
\hline
\end{tabular}

(c) applying a nontilted reference with tilted sample beam for testing plane mirrors (in the scanning optical head mode);

(d) utilizing a second optical head as a nontilted reference beam, similar to that one used on the LTP MF of NSRL, to overcome overlapping;

(e) employing a pentaprism scan, a good method of testing plane mirrors;

(f) alternatively switching on/off the sample and referencebeams during the scan (Figure 9(f)), with, for example, a variable polarizer.

Figure 10 compares measurements acquired by applying different nontilted reference methods with those from a tilted reference method. The height errors of two nontilted reference methods (a) and (b) are very close but very different from the tilted reference method (c).

4.3. Evolution of Scan Modes. The variance in scan modes is the outcome of evaluations of the profiler. After modifications, the patented LTP became the first commercial product, LTP II, which gained the 1993 R \& D 100 award. The LTP II applied the scanning optical head $(\mathrm{OH})$ in the tilted reference mode. While working with the Sincrotrone Trieste in 1995, I suggested utilizing the scanning pentaprism LTP (PPLTP) mode [5], an evolution that extends applications and improves the accuracy of tests for plane- and near-plane mirrors because a tilted reference beam is unnecessary. In 2006, the Nano-Optic-Measuring machine (NOM) applied 


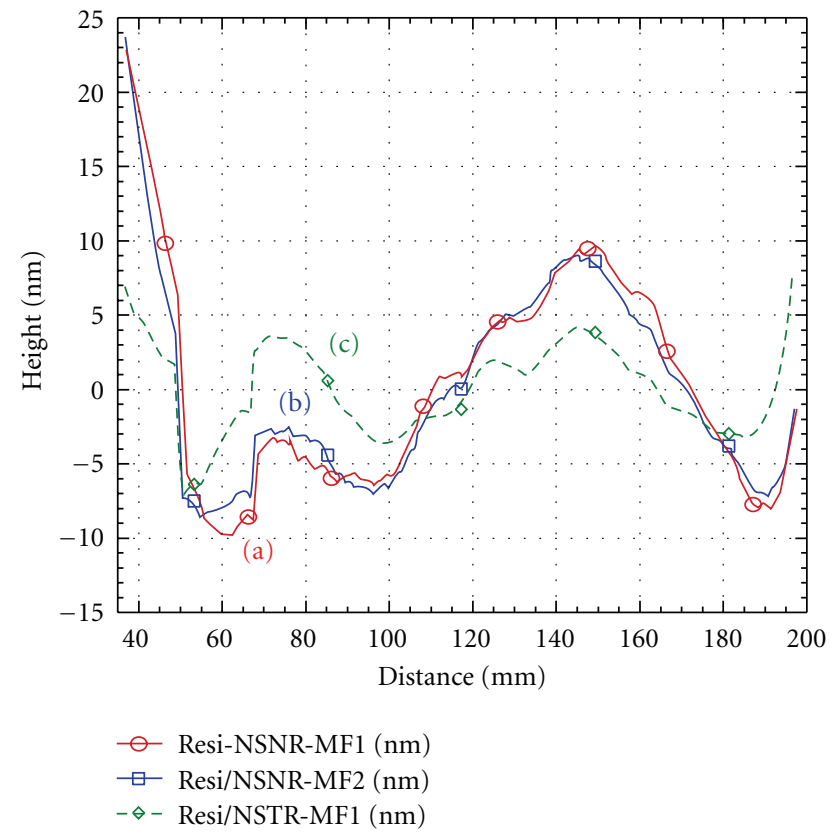

Figure 10: Comparison tests between tilted and nontilted reference methods. (a) Independent nontilted reference and nontilted sample. (b) Nontilted reference of second $\mathrm{OH}$ and nontilted sample. (c) Tilted reference and nontilted sample.

an autocollimator and adopted a series of error-reduction methods with a scanning pentaprism mode to enhance accuracy to the nanoradian level in small test angles. Adopting the suggested scanning $\mathrm{OH}$, combined with a nontilted reference mode, and improvements in the system's quality, will be another stage of evolution of the profiler towards reaching nanoradian accuracy in the larger slope test-range [35].

The finding in the slope error test of $1-2 \mu \mathrm{rad}$ rms described above caused by the BLM of $12 \mathrm{~mm}$, and the slope error with $\pm 1 \mathrm{~mm}$ (for $\pm 0.01 \mathrm{rad}$ angle test range) BLM in the scan $\mathrm{OH}+$ nontilted REF mode, can be estimated to drop down to the $0.2-0.3 \mu \mathrm{rad}$ rms level. In addition to maintaining a good measurement environment, if more efforts are made to better the quality of the optical components in the system and to correct residual system error via a single calibration curve, it likely will be possible to reach 100 nanoradian accuracy in \pm 0.01 rad test range.

Recently, the Physikalisch-Technische Bundesanstalt (PTB), described their new research in "Scanning deflectometric form measurement avoiding path-dependent angle measurement errors" [36]. Seemingly, this is a good method to reduce measurement error caused by the BLM. In the case of the PTB, the beam, reflected by a mirror setting on tilting stage for adjusting the mirror being test, can be considered as a fixed-distance tilting sampling beam to evaluate the mirror's slope; another beam is considered as nontilted reference. It is a similar way to reduce the BLM. However, for the PTB, the tilting stage must be especially precise.
Because the NSP uses pencil beam of $1-1.5 \mathrm{~mm}$ to scan the sample, the measurement's spatial frequency is larger than $1 \mathrm{~mm}$. Further improvements are necessary.

To the best of my knowledge, three scanning modes are used in different metrology laboratories around the world:

(a) Scanning pentaprism mode: NOM at BESSY II, Germany; Diamond-NOM at Diamond light source, UK; ESRF-LTP at ESRF, France; PPLTP at ELETTRA, Italy; PPLTP at Sprin-8, Japan; PPLTP at NSSRC, Taiwan; SSRF-LTP at SSRF, China; DLTP at ALS, USA; and, Soleil-LTP at Soleil, France;

(b) Scanning OH+ tilted ref: LTP III at BNL, USA; LTP VI at Swiss Light Source, Switzerland; LTP II at APS, USA; LTP VI at Deresbury; and, LTP II, Korea;

(c) Scanning $\mathrm{OH}+$ nontilted ref: LTP MFs at NSRL, China and BNL. There are several improvements needed; updating the best air bearing; increasing temperature stability; and, improving the quality of optical system. Thereafter, the LTP MFs will reach their potential nanoradian accuracy.

\section{Conclusion}

To reach nanoradian accuracy in the larger testing range for a surface profiler, the designer should consider eliminating or significantly reducing the beam's lateral motion thereby to decrease the impact caused by the imperfect optical components sited in the optical system's testing path. According to recent measurements, this slope error is about $1 \mu \mathrm{rad} \mathrm{rms}$ or up for current profilers in the test range of $\pm 0.02 \mathrm{rad}$. Adopting the suggested scanning $\mathrm{OH}$ mode, combined with a nontilted reference, along with improvements in the system's quality will be another stage of evolution of the new profiler towards reaching nanoradian accuracy in the larger slope test range of up to $\pm 0.01-0.015 \mathrm{rad}$.

Presently, most profilers operate in the scanning pentaprism mode (tilted scanning sample beam). They lack nanoradian accuracy for large angle test, and their final calibration is very difficult. Hence, further improvement is necessary.

\section{Acknowledgments}

The author would like to thank Dr. Daniele Cocco of Sincrotrone Trieste for helpful discussion on the subject of this paper. This paper has been authored by Brookhaven Science Associates, LLC under Contract no. DE-AC0298CH10886 with the U.S. Department of Energy. The United States Government retains, and the publisher, by accepting the article for publication, acknowledges, a worldwide license to publish or reproduce the published form of this paper, or allow others to do so, for the United States Government purposes. This research was sponsored by the U. S. Department of Energy. 


\section{References}

[1] H. Mimura, S. Handa, T. Kimura et al., "Breaking the $10 \mathrm{~nm}$ barrier in hard-X-ray focusing," Nature Physics, vol. 6, no. 2, pp. 122-125, 2010.

[2] D. M. Sykora, "A model for cavity induced errors with wavefront slope in high accuracy spherical fizeau metrology," in Proceedings of the Optical Fabrication and Testing Conference (OFT '08), Rochester, NY, USA, October 2008.

[3] R. N. Youngworth and B. D. Stone, "Simple estimates for the effects of mid-spatial-frequency surface errors on image quality," Applied Optics, vol. 39, no. 13, pp. 2198-2209, 2000.

[4] D. M. Aikens, J. E. Degroote, and R. N. Youngworth, "Specification and control of mid-spatial frequency wavefront errors in optical systems," in Proceedings of the Optical Fabrication and Testing Conference (OFT '08), Rochester, NY, USA, October 2008.

[5] C. Pruss, E. Garbusi, and W. Osten, “Testing aspheres," Optics and Photonics News, vol. 19, no. 4, pp. 24-29, 2008.

[6] P. Murphy, G. Devries, J. Fleig, G. Forbes, A. Kulawiec, and D. Miladinovic, "Measurement of high-departure aspheric surfaces using subaperture stitching with variable null optics," in Optical Manufacturing and Testing VIII, vol. 74260 of Proceedings of SPIE, August 2009.

[7] http://www.qedmrf.com/metrology/ssi-technology/, 2011.

[8] M. Schulz, A. Wiegmann, A. Márquez, and C. Elster, "Optical flatness metrology: 40 years of progress," Optica Pura Aplicada, vol. 41, no. 4, pp. 325-331, 2008.

[9] K. Becker and E. Heynacher, "M400— a coordinate measuring machine with $10 \mathrm{~nm}$ resolution," in Optical Metrology for Precision Machining, vol. 802 of Proceedings of SPIE, pp. 209216, 1987.

[10] A. Lahsen, B. Michael, and S. Deming, "Development of a linear stitching interferometric system for evaluation of very large X-ray synchrotron radiation substrates and mirrors," in Proceedings of the 8th International Conference on Synchrotron Radiation Instrumentation, vol. 705 of American Institute of Physics Conference Proceedings, pp. 851-854, 2004.

[11] P. François, T. Muriel, B. Sylvain, and R. Amparo, "An LTP stitching procedure with compensation of instrument errors: comparison of SOLEIL and ESRF results on strongly curved mirrors," Nuclear Instruments and Methods in Physics Research Section A, vol. 616, no. 2-3, pp. 207-211, 2010.

[12] P. Z. Takacs and S. N. Qian, "Design of a long trace surface profiler," in Metrology: Figure and Finish, B. E. Truax, Ed., vol. 749 of Proceedings of SPIE, pp. 59-64, 1987.

[13] P. Z. Takacs and S. N. Qian, "Surface profile interferometer," US patent 4884697, December 1989.

[14] K. V. Bieren, "Pencil beam interferometer for aspherical potical surfaces," in Laser Diagnostics, vol. 343 of Proceedings of SPIE, pp. 101-108, 1982.

[15] S. N. Qian, W. Jark, and P. Takacs, "The penta-prism LTP: a long-trace-profiler with stationary optical head and moving penta prism," Review of Scientific Instruments, vol. 66, no. 3, pp. 2562-2569, 1995.

[16] S. N. Qian, W. Jark, P. Takacs, K. Randell, and W. Yun, "Insitu surface profiler for high heat load mirror measurement," Optical Engineering , vol. 34, no. 2, pp. 396-402, 1995.

[17] S. N. Qian and P. Z. Takacs, "Portable long trace profiler: concept and solution," Review of Scientific Instruments, vol. 72, no. 8, pp. 3198-3204, 2001.

[18] H. Z. Li, X. J. Li, M. W. Grindel, and P. Z. Takacs, "Measurement of X-ray telescope mirrors using a vertical scanning long trace profiler," Optical Engineering, vol. 35, no. 2, pp. 330-338, 1996.

[19] S. N. Qian and P. Z. Takacs, "Design of multiple-function long trace profiler," Optical Engineering, vol. 46, no. 4, Article ID 043602, 9 pages, 2007.

[20] S. N. Qian, W. Jark, A. Gambitta, F. Mazzolini, and A. Savoia, "Precise measuring method for detecting the in situ distortion profile of a high-heat-load mirror for synchrotron radiation by use of a pentaprism long trace profiler," Applied Optics, vol. 36, no. 16, pp. 3769-3775, 1997.

[21] S. N. Qian, D. Arena, J. Dvorak, and K. Qian, "Dynamic monitoring of grating angle at national synchrotron light source," Optical Engineering, vol. 48, no. 11, Article ID 113603, 2009.

[22] J. Susini, R. Baker, and A. Vivo, "Optical metrology facility at the ESRF," Review of Scientific Instruments, vol. 66, no. 2, pp. 2232-2234, 1995.

[23] M. Thomasset, S. Brochet, and F. Polack, "Latest metrology results with the SOLEIL synchrotron LTP," in Advances in Metrology for X-Ray and EUV Optics, vol. 5921 of Proceedings of SPIE, San Diego, Calif, USA, August 2005.

[24] D. Zeng, T. Xiao, G. Du et al., "New long trace profiler based on phase plate diffraction for optical metrology of SSRF," Review of Scientific Instruments, vol. 77, no. 9, Article ID 093305, 5 pages, 2006.

[25] F. Siewert, T. Noll, T. Schlegel, T. Zeschke, and H. Lammert, "The nanometer optical component measuring machine: a new sub-nm topography measuring device for X-ray optics at BESSY," in Proceedings of the 8th International Conference on Synchrotron Radiation Instrumentation, vol. 705 of American Institute of Physics Conference Proceedings, pp. 847-850, May 2004.

[26] F. Siewert, R. Follath, T. Noll et al., "0.05 $\mu \mathrm{rad}$ accuracy metrology for a new generation of reflective SR-optics," http://goldberg.lbl.gov/MetrologyWorkshop07/originals/ Siewert_ALS_UM.pdf.

[27] S. Alcock, K. Sawhney, S. Scott et al., "The diamond-nom: a non-contact profiler capable of characterizing optical figure error with sub-nanometre repeatability," Nuclear Instruments and Methods in Physics Research Section A, vol. 616, no. 2-3, pp. 224-228, 2010.

[28] M. Schulz, G. Ehret, M. Stavridis, and C. Elster, "Concept, design and capability analysis of the new deflectometric flatness reference at PTB," Nuclear Instruments and Methods in Physics Research Section A, vol. 616, no. 2-3, pp. 134-139, 2010.

[29] A. Wiegmann, M. Schulz, and C. Elster, "Improving the lateral resolution of a multi-sensor profile measurement method by non-equidistant sensor spacing," Optics Express, vol. 18, no. 15, pp. 15807-15819, 2010.

[30] http://www.ptb.de/cms/en/fachabteilungen/abt8/fb-84/ag -842formmessung-842.html, 2011.

[31] S. N. Qian and K. Qian, "Study and considerations of nanometer and nano-radian surface profiler," in Proceedings of the 5th International Symposium on Advanced Optical Manufacturing and Testing Technologies: Optical Test and Measurement Technology and Equipment, vol. 7656 of Proceddings of SPIE, Dalian, China, 2010.

[32] F. Siewert, J. Buchheiim, and T. Zeschke, "Characterization and calibration of 2nd generation slope measuring profiler," Nuclear Instruments and Methods in Physics Research Section A, vol. 616, no. 2-3, pp. 119-127, 2010. 
[33] P. Z. Takacs and S. N. Qian, "The long trace profiler after 20 years - what works and what doesn't work," in Optics and Photonics, Proceedings of SPIE, San Diego, Calif, USA, 2010.

[34] J. L. Kirschman, E. E. Domning, G. Y. Morrison, B. V. Smith, and V. V. Yashchuk, "Precision tiltmeter as a reference for slope measuring instruments," in Advances in Metrology for X-Ray and EUV Optics II, vol. 6704 of Proceedings of SPIE, San Diego, Calif, USA, 2007.

[35] S. Qian, K. Qian, Y. Hong, L. Sheng, T. Ho, and P. Z. Takacs, "Systematic error reduction: non-tilted reference beam method for long trace profiler," in Advances in Metrology for X-Ray and EUV Optics II, vol. 6704 of Proceedings of SPIE, San Diego, Calif, USA, August 2007.

[36] M. Schulz, G. Ehret, and A. Fitzenreiter, "Scanning deflectometric form measurement avoiding path-dependent angle measurement errors," Journal of the European Optical Society, vol. 5, Article ID 10026, 2010. 

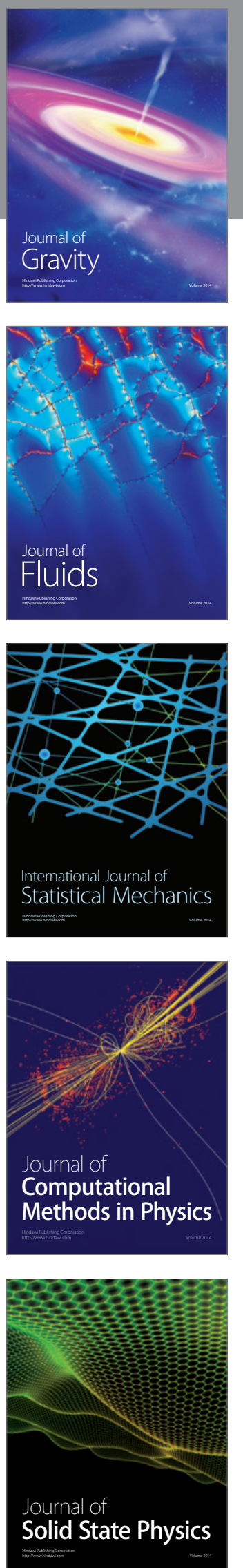

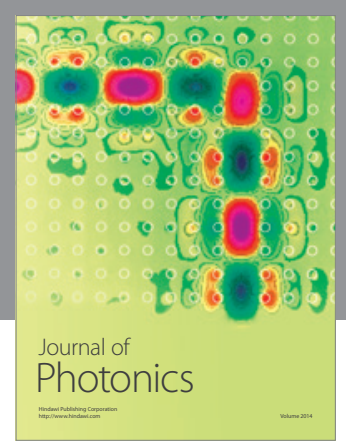

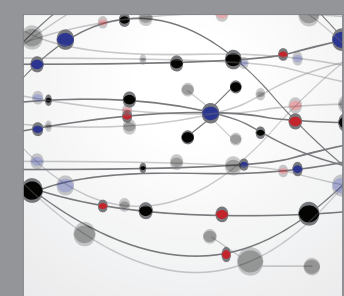

The Scientific World Journal
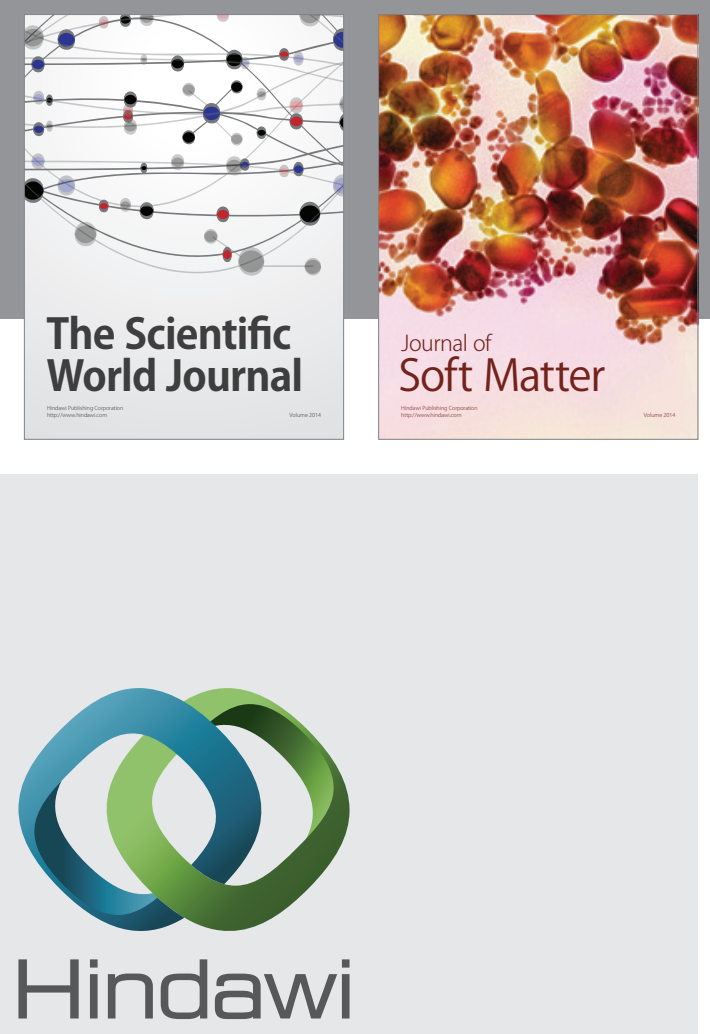

Submit your manuscripts at

http://www.hindawi.com
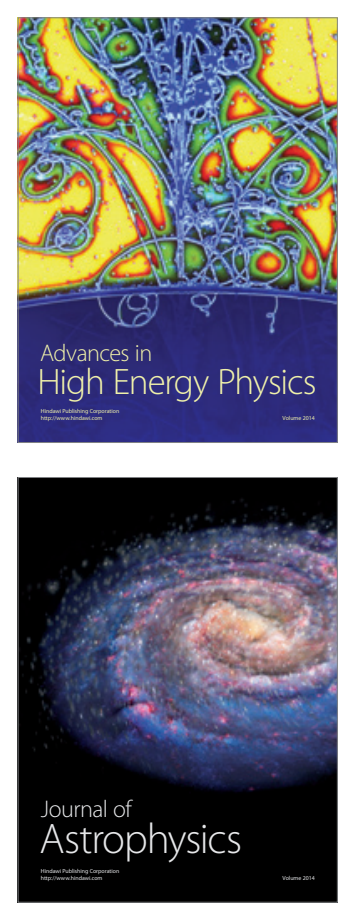
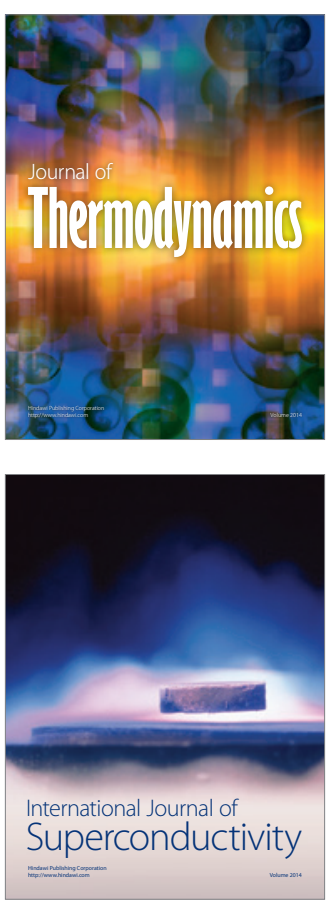
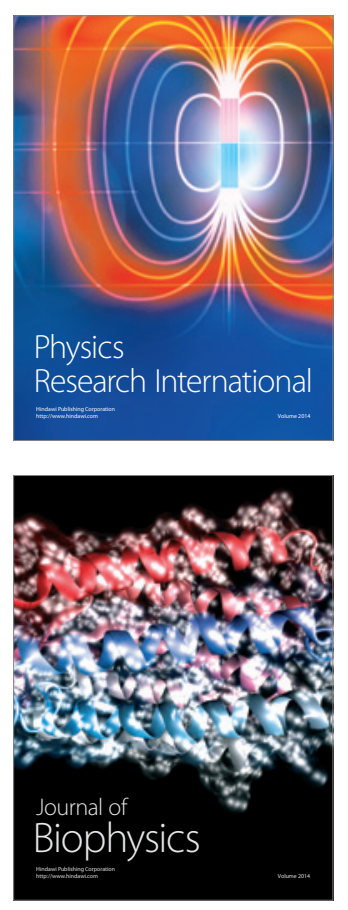
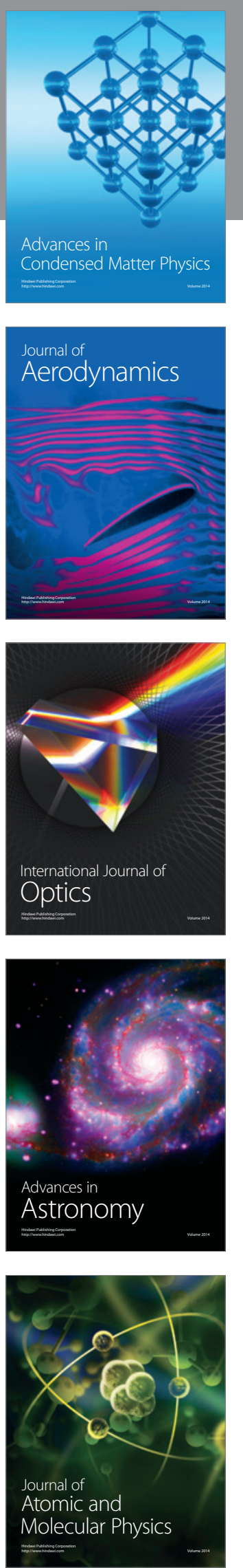